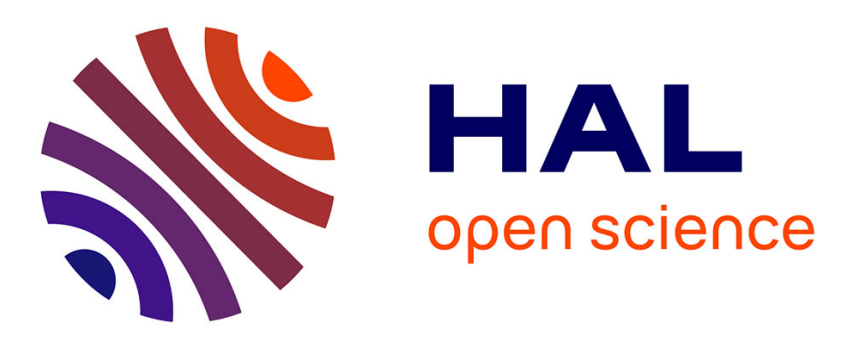

\title{
Determination of photo-induced Seebeck coefficient for hot carrier solar cell applications
}

\author{
Hamidreza Esmaielpour, Daniel Suchet, Laurent Lombez, Amaury Delamarre, \\ Soline Boyer-Richard, Alexandre Beck, Alain Le Corre, Olivier Durand, \\ Jean-Francois Guillemoles
}

\section{To cite this version:}

Hamidreza Esmaielpour, Daniel Suchet, Laurent Lombez, Amaury Delamarre, Soline Boyer-Richard, et al.. Determination of photo-induced Seebeck coefficient for hot carrier solar cell applications. 47th Photovoltaic Specialists Conference (PVSC 2020), Jun 2020, Calgary (virtual), Canada. pp.0747-0751, 10.1109/PVSC45281.2020.9300544 . hal-03223077

\section{HAL Id: hal-03223077 \\ https://hal.science/hal-03223077}

Submitted on 26 Nov 2021

HAL is a multi-disciplinary open access archive for the deposit and dissemination of scientific research documents, whether they are published or not. The documents may come from teaching and research institutions in France or abroad, or from public or private research centers.
L'archive ouverte pluridisciplinaire HAL, est destinée au dépôt et à la diffusion de documents scientifiques de niveau recherche, publiés ou non, émanant des établissements d'enseignement et de recherche français ou étrangers, des laboratoires publics ou privés. 


\section{Determination of photo-induced Seebeck coefficient for hot carrier solar cell applications}

\author{
Hamidreza Esmaielpour \\ Institut Photovoltaique d'Ile de \\ France (IPVF), UMR \\ 9006,Palaiseau, France \\ hamidreza.esmaielpour@ipvf.fr \\ Soline Boyer-Richard \\ Univ Rennes, INSA Rennes, \\ CNRS, Institut FOTON - UMR \\ 6082, Rennes, France \\ soline.boyer@insa-rennes.fr
}

\author{
Daniel Suchet \\ Ecole Polytechnique, IPVF \\ UMR 9006, Palaiseau, France \\ daniel.suchet@polytechnique.org
}

\author{
Alexandre Beck \\ Univ Rennes, INSA Rennes, \\ CNRS, Institut FOTON - UMR \\ 6082, Rennes, France \\ alexandre.beck@insa-rennes.fr
}

\author{
Laurent Lombez \\ CNRS- IPVF, UMR 9006, \\ Palaiseau, France \\ laurent.lombez@cnrs.fr
}

\author{
Alain Le Corre \\ Univ Rennes, INSA Rennes, \\ CNRS, Institut FOTON - \\ UMR 6082, Rennes, France \\ alain.le-corre@insa-rennes.fr
}

\author{
Amaury Delamarre \\ Centre for NanoSci. and NanoTech, \\ CNRS, Palaiseau, France \\ amaury.delamarre@universite-paris- \\ saclay.fr \\ Olivier Durand \\ Univ Rennes, INSA Rennes, CNRS, \\ Institut FOTON - UMR 6082, \\ Rennes, France \\ olivier.durand@insa-rennes.fr
}

\author{
Jean-François Guillemoles \\ CNRS-IPVF, UMR 9006, Palaiseau, France \\ jean-francois.guillemoles@cnrs.fr
}

\begin{abstract}
Determination of Seebeck coefficient is a practical technique to investigate the direct conversion of hot carrier energy to electric voltage. However, this study is challenging, especially in nanostructured materials using traditional measurements via heaters and electric contacts. Here, we investigate photo-induced Seebeck coefficient of InGaAs multi-quantum-well structure via a contact-less measurement (photoluminescence spectroscopy). We have determined thermodynamic properties of hot carriers via fitting the emitted photoluminescence spectra with the generalized Planck's law. We have observed a linear dependence between the gradient of carrier temperature and the quasi-Fermi level splitting of photo-generated hot carriers at various lattice temperatures, which is associated with thermoelectric effects in the system.
\end{abstract}

Keywords-Hot carrier extraction, Thermoelectric effect, Inplane photo-Seebeck coefficient, Photoluminescence spectroscopy.

\section{INTRODUCTION}

Hot carrier solar cells are one of the third-generation photovoltaic devices, which have potential to enhance the efficiency of single junction solar cells beyond the fundamental limit (33\%). [1] Two of the main challenges in this technology are: 1) inhibiting hot carrier thermalization, and 2) fast extraction of hot carriers via energy selective contacts. In last few years, there has been significant progress in designing efficient hot carrier absorbers with limited thermalization loss using nanostructured materials. [2]-[4] However, there are still problems regarding the efficient extraction of hot carriers from these designs. Therefore, an improvement in energy selective contacts will be significant in this technology.

Recently, extraction of hot carrier energies via thermoelectric effects has attracted a lot of interest. [5] In this technique, the gradient of carrier temperature between the hot absorber and the cold contact creates a driving force for the direct conversion of heat to electricity, which is known as the Seebeck effect. [5] Traditional experiments for determination of Seebeck coefficients require local heating and simultaneously measuring voltage, which is challenging and electrical artifacts in the system can add uncertainties to the results. [6] Recently, a contact-less measurement for determination of Seebeck coefficients has been reported by Gibelli et al. [6]-[7] using photoluminescence (PL) spectroscopy, which can eliminate issues regarding traditional methods. Here, we investigate the in-plane Seebeck coefficient of an InGaAs multi-quantum well (MQW) structure using PL spectroscopy at various excitation powers and lattice temperatures.

\section{EXPERMENTS AND DISCUSSIONS}

PL spectroscopy of $\operatorname{In}_{0.53} \mathrm{Ga}_{0.47} \mathrm{As}$ MQW structure is studied under continuous wave $442 \mathrm{~nm}$ laser excitation at room temperature. The InP lattice-matched MQW structure comprises five un-doped InGaAs quantum wells $(5.5 \mathrm{~nm})$ and InGaAsP barriers $(10 \mathrm{~nm})$. The $\mathrm{QW}$ region (active region) is isolated by InP cladding layers, in order to accumulate photo-generated particles within the active region.

A hyperspectral image of the emitted PL spectrum by the sample at room temperature is shown in Figure 1 (a). The image shows a top view of the 3D image and the vertical and horizontal axes indicate the $x-y$ plane of the sample. The out-of-plane (or " $z$ ") axis indicates the emission intensity and the scale bar on the top of the image defines the range of the absolute calibrated photon fluxes.

It is observed that the spatial distribution of the emitted PL spectrum is circular (or symmetric) around the PL spot. This image shows how photo-generated particles are distributed spatially in the planar structure. It is expected particles (electrons and holes) at the center of the spot to be at elevated temperatures compared with particles at larger distances. Thermal gradient between hot to cold regions creates a diffusion current, which is described by thermoelectric effects. Diffusion of hot carriers in the sample due to thermal gradient induces an electric potential across the hot to cold regions and the strength of this effect is 
determined by the Seebeck coefficient $(\alpha)$, which is given by: [6]

$\alpha=-\frac{\Delta \mu}{q \Delta T}$,

where $\mathrm{q}$ is coulomb charge, $\Delta \mu$ and $\Delta \mathrm{T}$ are the gradient of the quasi-Fermi level splitting and the temperature difference of particles across the two regions, respectively. In the PL spectroscopy (in the present case), a simultaneous diffusion of electrons and holes in a temperature gradient is measured, which determines the ambipolar Seebeck coefficient. Thermal gradient in the system causes hot carrier transport in both in-plane and cross-plane (across the interface between hot and cold layers) directions. Hyperspectral luminescence imaging provides information regarding in-plane photo-Seebeck effects via
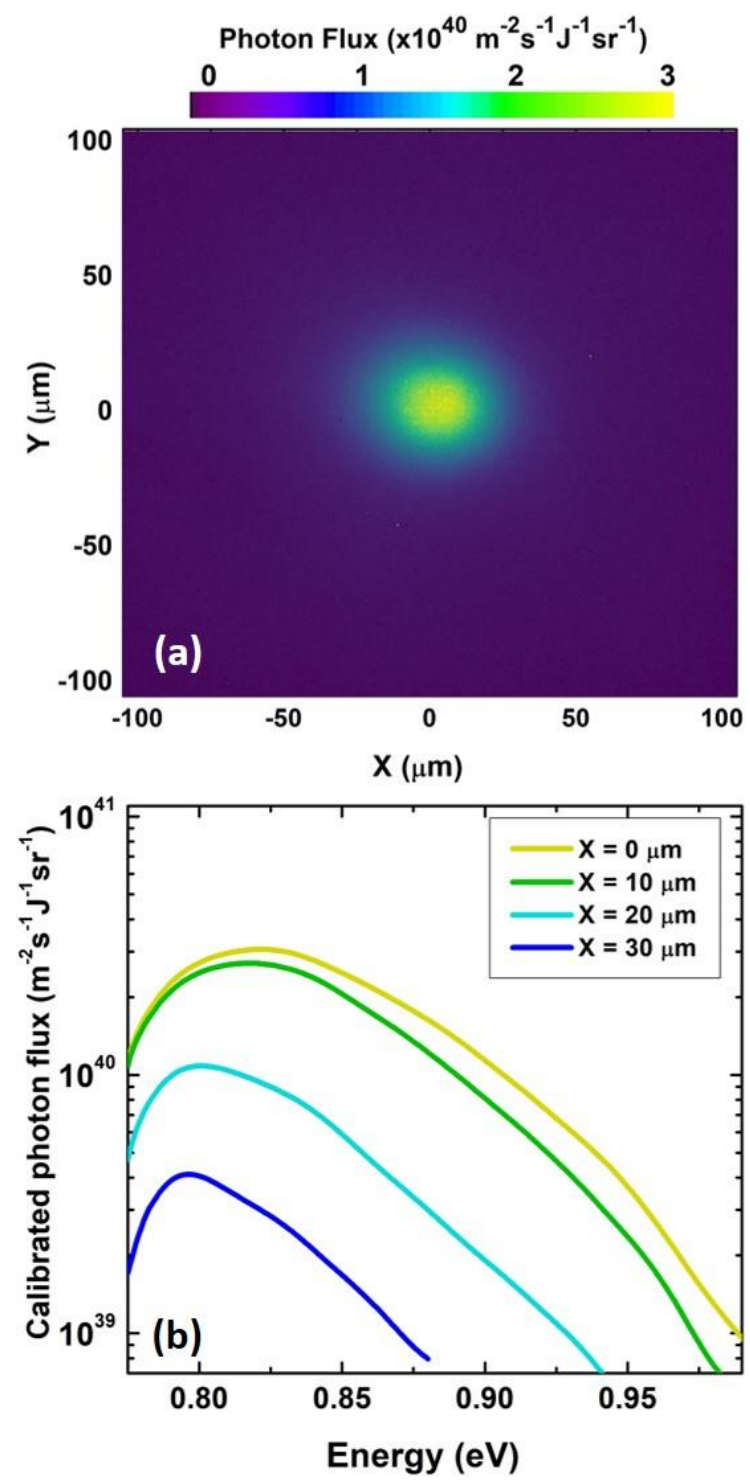

Figure 1. (a) Hyperspectral luminescence imaging of the emitted PL spectrum from the InGaAs MQW structure. (b) Absolutely calibrated PL spectra of the MQW structure at different distances from the PL spot. showing the spatial distribution of hot carriers in the MQW structure. In addition, it would be possible to investigate the cross-plane photo-Seebeck effect, if there is PL emission from both materials (the QW and the barrier) across the interface. This is an important study, especially for hot carrier extraction applications.

Calibrated PL spectra versus photon energy at various distances from the PL spot are plotted in Figure 1 (b). It is observed that as the distance from the PL spot increases, the broadening of the high energy side of the PL spectra becomes smaller, which is indicative of less effects associated with hot carriers and occupation of excited states.

It is possible to determine thermodynamic properties of emitting carriers such as, temperature and quasi-Fermi level splitting, via fitting the emitted PL spectra with the generalized Planck's law, as described by: [2]

$I_{P L}(E)=\frac{2 \pi A(E)(E)^{2}}{h^{3} c^{2}}\left[\exp \left(\frac{E-\Delta \mu}{k_{B} T}\right)-1\right]^{-1}$,

where " $I_{P L}$ " is the PL intensity, " $h$ " the Planck's constant, " $c$ " the speed of light, " $k_{B}$ " the Boltzmann's constant, and " $A$ " the absorptivity of the sample. Due to the existence of multiple transitions in the system associated with the occupation of excited states, determination of carrier temperature via simply applying a linear fit to the high energy side of PL spectra, which is the usual method, will lead to erroneous results. [8]

Another technique to determine carrier temperature is to fit the full PL spectrum with the generalized Plank's law. In this method, it is required to model the energy dependent absorptivity of the sample via considering different optical transitions in the system. This is a robust technique and it is possible to include quantum confinement effects such as, multiple transitions from discrete energy levels and band filling, in order to have a better estimate regarding the actual hot carrier properties. This method also provides a better insight about thermodynamic and optical properties of the system. Although, this technique can improve the precision of carrier temperature determination, it adds complications to the analysis, especially if there are multiple discrete energy levels in the system. [8],[9] It is also slow and makes it difficult to treat hyperspectral images with large number of spectra (there are $>10^{5}$ spectra in the image shown in Figure 1 (a)!).

The third technique for carrier temperature determination is PL ratio method, which has a much more precision compared with the linear fitting method and less complications than the full PL spectrum fitting. In this method, carrier temperature is determined via dividing a PL spectrum by a reference one, as described by:

$\ln \left(\frac{I_{2}}{I_{1}}(E)\right) \approx\left[\frac{\mu_{2}}{k_{B} T_{2}}-\frac{\mu_{1}}{k_{B} T_{1}}\right]+\left[\frac{1}{k_{B} T_{1}}-\frac{1}{k_{B} T_{2}}\right] \times E$,

where the index " 1 " is associated with the reference PL spectrum with a well-defined carrier temperature and " 2 " is related to the spectrum with unknown " $T$ " and " $\mu$ ". This equation is valid under the Boltzmann approximation and in the 

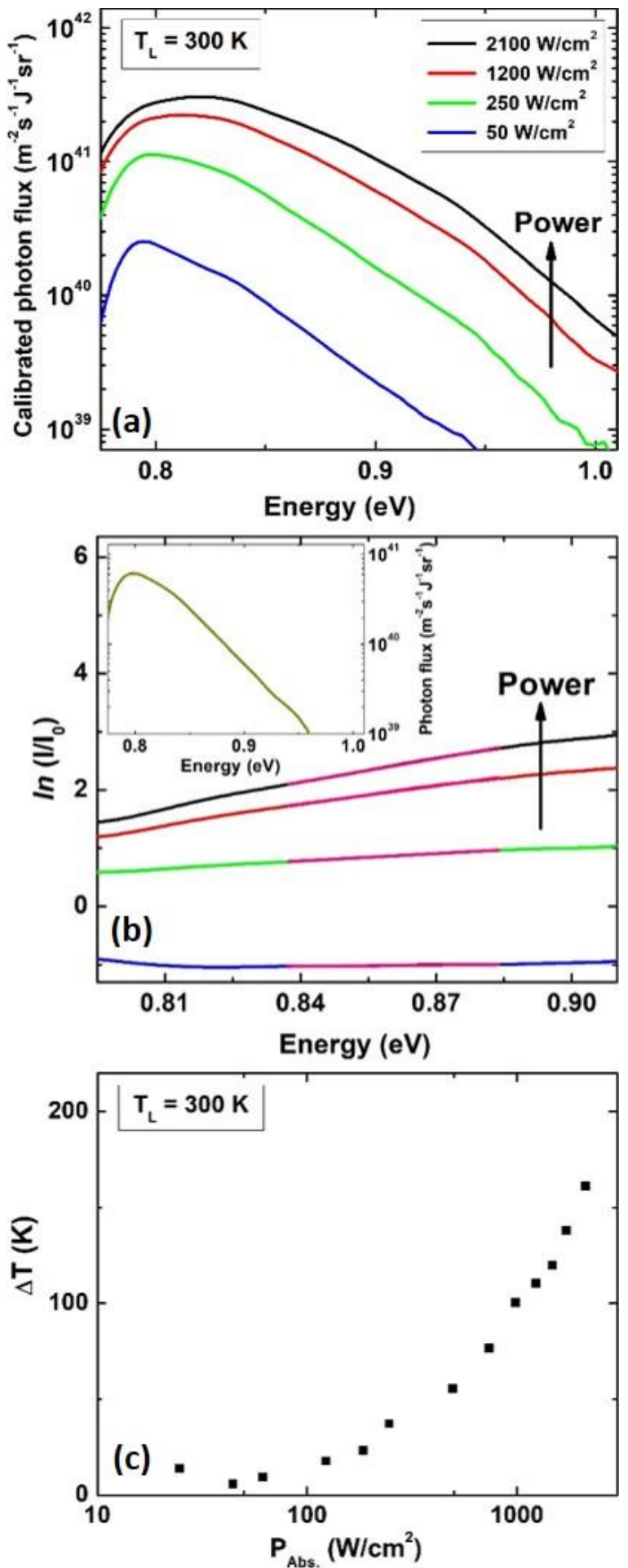

Figure 2. (a) Calibrated PL spectra of the InGaAs MQW structure at canter of the PL spot under various excitation powers. (b) The results of the PL ratio method for the PL spectra shown in panel (a). The solid pink lines show the energy regions, where carrier temperatures are determined from the slopes. The inset of the graph indicates the PL spectrum emitted by thermalized carriers at $15 \mu \mathrm{m}$ away from the concentrated laser. This spectrum is considered as the reference spectrum for the PL ratio method. (c) Excitation power dependent $\Delta T$ at $300 \mathrm{~K}$. range of energies, where the absorptivity of both PL spectra is similar. As a result, the absorptivity term will be removed, see equation (3), and further complications regarding this term will be avoided. Another advantage of this technique is that it can remove the effects of optical artifacts from the PL spectra, induced by optical characterization devices, and improve estimated carrier temperatures.

The slope in equation (3) is proportional with the temperature of emitting particles. In this method, it is essential to use a reference spectrum with a well-defined carrier temperature $\left(T_{1}\right)$, because both the absolute value and the trend of change in carrier temperature depend on the temperature of the reference PL spectrum. Carrier temperature in PL ratio method is determined by:

$$
T_{2}=\frac{T_{1}}{1-k_{B} T_{1} \times(\text { slope })} .
$$

There are different ways to find a reference spectrum with a well-defined carrier temperature. One way is to use a PL spectrum emitted under a very weak excitation power. At this condition, due to weak excitation, the carrier temperature of the emitting particles will be at the same temperature as the crystal lattice. Another method is to utilize the PL spectrum emitted from a region on the sample far from the concentrated excitation laser. Due to the short hot carrier diffusion length $(<0.5 \mu \mathrm{m})$ in semiconductors, [10]-[11] it is possible to select a PL spectrum from a region far from the concentrated light. This data can be provided by an image from a hyperspectral luminescence system.

Absolutely calibrated PL spectra of InGaAs MQW structure at room temperature under four different excitation powers are plotted in Figure 2 (a). The PL spectra in this figure are emitted from the center of PL spots. It is observed that by increasing the excitation power, the slopes at high energy side of the PL spectra become shallower. Figure 2 (b) shows the results of the PL ratio method for the same PL spectra as Figure 2 (a). The range of energies, where the slops are determined for each spectrum is shown by solid pink lines in the graph. The reference PL spectrum, which is applied for this analysis, is plotted in the inset of Figure 2 (b). The reference spectrum is taken from a region on the sample at a distance $15 \mu \mathrm{m}$ from the concentrated laser light. It is observed that there is a linear dependence between $\ln \left(I / I_{0}\right)$ and the photon energy, which confirms that the absorptivity of all spectra at the range of studied energies is the same. The slope of each spectrum is proportional with the carrier temperature, see equation (3) and (4), and the Y-intercept is proportional with the quasi-Fermi level splitting. The increase in the slope in this figure is indicative of an increase in carrier temperature as a function of excitation power.

The results of excitation power dependent temperature difference, $\Delta T$, (temperature difference between hot carriers and lattice) at $300 \mathrm{~K}$ are plotted in Figure 2 (c). It is seen that up to $100 \mathrm{~W} / \mathrm{cm}^{2}$ the change in $\Delta T$ is negligible, which is indicative of an insufficient excitation power for the creation of a stabilized hot carrier population in the system. By increasing the excitation power above the threshold value, an increase in $\Delta T$ is observed.

Figure 3 (a) shows the spatial distribution of $\Delta T$ as a function of distance from the PL spot at various lattice temperatures 
under $470 \mathrm{~W} / \mathrm{cm}^{2}$ excitation power ( $\left.4700 \mathrm{Suns}\right)$. It is seen that at the center of the PL spot, $\Delta T$ is large, then it reduces at larger distances. The drop of $\Delta T$ continues as a function of distance until it becomes zero. This effect indicates that at large distances, carriers are completely thermalized. Moreover, Figure 3 (a) shows that there is an increase in $\Delta T$ as a function of lattice temperature, which is an unusual behavior. The origin of this effect is under investigation. In general, by increasing the lattice temperature, the density of phonons in the system becomes larger; therefore, it is expected to observe larger phononmediated thermalization rates for hot carriers, or lower $\Delta T$ values.
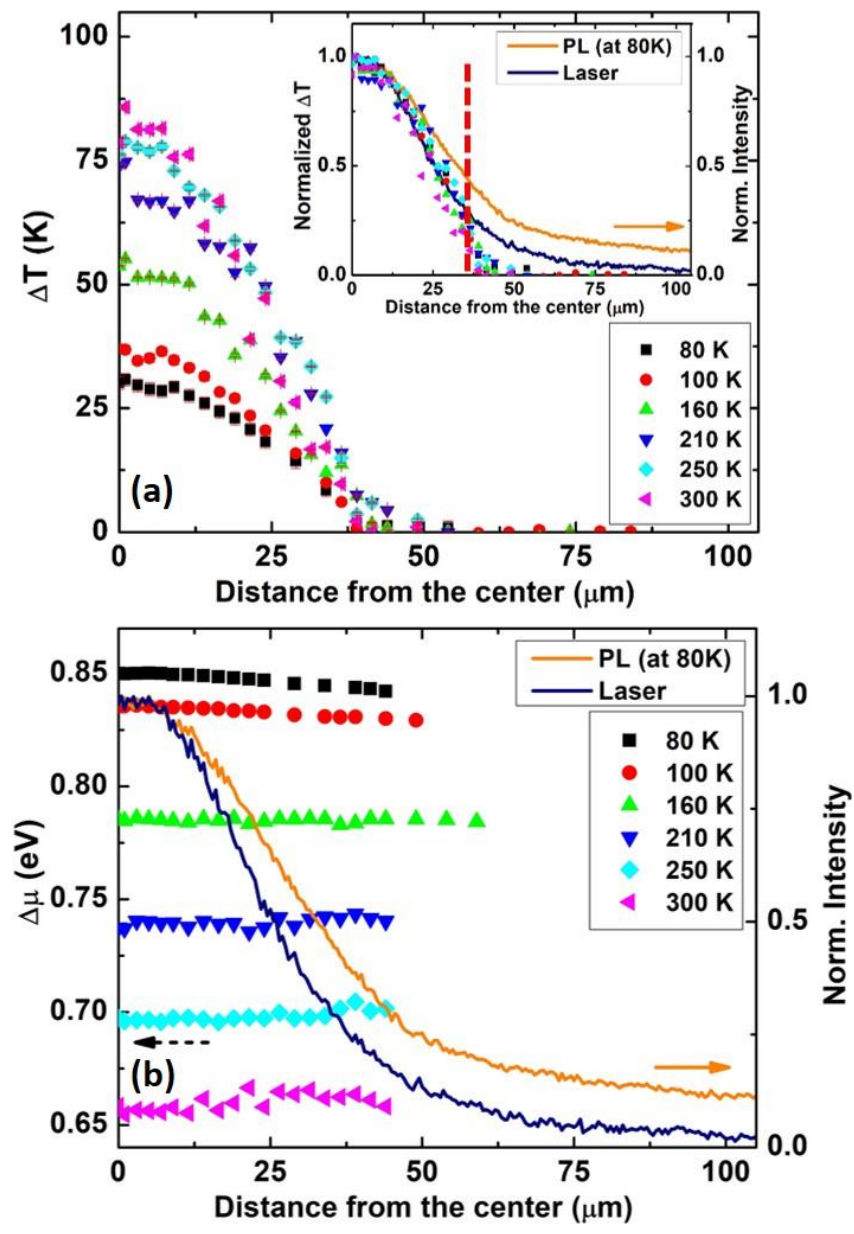

Figure 3. (a) Temperature difference $(\Delta T)$ versus distance from the laser spot at various lattice temperatures. The inset shows the normalized $\Delta T$ as a function of distance. Solid orange and dark lines indicate the spatial distribution of the emitted PL at $80 \mathrm{~K}$ and the excitation laser. The red dashed line shows the region, where beyond that point hot carrier thermalization increases. (b) Quasi-Fermi level splitting $(\Delta \mu)$ as a function of distance. The orange and dark blue lines (similar to the inset of the panel (a)) show the spatial distribution of the emitted PL spectrum and the laser excitation.

The inset of Figure 3 (a) shows the change of normalized $\Delta T$ in the main panel versus distance. The spatial distribution of the normalized concentrated laser light and normalized emitted PL spectrum at $80 \mathrm{~K}$ are plotted by dark blue and orange colors, respectively. Due to the similarity in the spatial distribution of the emitted PL spectra at various lattice temperatures, the spectrum at one lattice temperature $(80 \mathrm{~K})$ is plotted. It is observed that there is a similar behavior for the change in $\Delta T$ as a function of distance, which mainly follows the spatial distribution of the laser light. This behavior continues up to around $\Delta x=35 \mu \mathrm{m}$, see the red dotted line, then above that, there is a fast drop in $\Delta T$, indicating a fast thermalization rate beyond that distance. As shown in the inset, although there is still laser illumination in the system above $\Delta x>35 \mu \mathrm{m}$, the amount of local excitation power is not enough in order to create a stabilized hot carrier distribution. In addition, the comparison between the laser and the PL spatial distributions indicates that there are emitting (photo-generated) particles with zero $\Delta T$ values (thermalized particles) at regions even under weak excitation intensities. This effect is attributed to the lateral diffusion of particles in the system after excitation.

Figure 3 (b) shows the temperature dependent quasi-Fermi splitting as a function of distance. The initial quasi-Fermi level splitting is calculated from the generalized Plank's law and the absorptivity of the MQW structure is determined by transfer matrix analysis. It is observed that by increasing the lattice temperature, there is a drop in $\Delta \mu$, which is due to the increase in the non-radiative recombination and the shrinkage of the band gap at elevated lattice temperatures. In addition, it is seen that the change of $\Delta \mu$ as a function of distance is negligible, especially at elevated lattice temperatures. The origin of this effect is still under investigation.

The photo-Seebeck coefficient of the MQW structure can be determined via studying the change of the local $\Delta \mu$ as a function of carrier temperature. A linear dependence between $\Delta \mu$ and carrier temperature is observed, and the slope of this trend defines the in-plane Seebeck coefficient of the sample. In addition, it is seen that the sign of the Seebeck coefficient changes at elevated lattice temperatures. This effect can be related to the violation of some assumptions made for the PL ratio analysis, especially under high illumination when the absorptivity of the sample changes as a function of excitation power. Further investigations using the full fitting method are required in order to find the origin of this mechanism.

\section{CONCLUSION}

We have studied the spatial distribution of hot carriers in the InGaAs MQW structure via hyperspectral luminescence imaging at various lattice temperatures. The temperature and the quasi-Fermi level splitting of photo-generated carriers are determined via fitting the emitted PL spectra with the generalized Planck's law. The advantage of this contact-less measurement is its capability to eliminate artifacts associated with electric contacts. The results indicate that at the center of the PL spot carrier temperature is large and as the distance from the spot increases, a drop in $\Delta T$ is observed. In addition, it is seen that at above $35 \mu \mathrm{m}, \Delta T$ is zero, which indicates that photo-generated carriers above this distance are completely thermalized and have the same temperature as the lattice. A linear dependence between $\Delta \mu$ and carrier temperature is observed and the slope of the graph defines the magnitude of the in-plane Seebeck coefficient of the sample. Further 
investigations will be performed at various lattice temperatures and excitation powers in order to achieve a better understanding about the behavior of hot carriers in the MQW structure.

\section{REFERENCES}

[1] G. Conibeer, et al. "Progress on hot carrier cells," Solar Energy Materials and Solar Cells, 93 (6-7), 2009, pp.713-719.

[2] A. Le Bris, et al. "Thermalisation rate study of GaSb-based heterostructures by continuous wave photoluminescence and their potential as hot carrier solar cell absorbers." Energy \& Environmental Science 5(3), 2012, pp. 6225-6232.

[3] L. C. Hirst, H. Fujii, Y. Wang, M. Sugiyama, and N.J. Ekins-Daukes, "Hot carriers in quantum wells for photovoltaic efficiency enhancement," IEEE Journal of Photovoltaics, 4(1), 2013, pp.244-252.

[4] H. Esmaielpour, et al. "Suppression of phonon-mediated hot carrier relaxation in type-II InAs/AlAsSb quantum wells: a practical route to hot carrier solar cells," Prog. in PV: Res and Appl, 24(5), 2016, pp.591-599.

[5] S.C. Limpert, and S.P. Bremner, "Hot carrier extraction using energy selective contacts and its impact on the limiting efficiency of a hot carrier solar cell," Applied Physics Letters, 107(7), 2015, p.073902.

[6] F. Gibelli, et al. "Optical imaging of light-induced thermopower in semiconductors," Physical Review Applied, 5(2), 2016, p.024005.

[7] F. Gibelli, et al. "Reply to "Comment on "Optical Imaging of LightInduced Thermopower in Semiconductors"'." Physical Review Applied 9.5 (2018): 058001.

[8] H. Esmaielpour, et al. "Advanced analysis for hot-carriers photoluminescence spectrum." Physics, Simulation, and Photonic Engineering of Photovoltaic Devices IX. Vol. 11275. International Society for Optics and Photonics, 2020.

[9] D. T. Nguyen, et al. "Quantitative experimental assessment of hot carrierenhanced solar cells at room temperature." Nature Energy 3(3), 2018, pp. 236-242.

[10] P. Yuan, et al. "The hot carrier diffusion coefficient of sub-10 $\mathrm{nm}$ virgin MoS 2: uncovered by non-contact optical probing." Nanoscale 9(20), 2017, pp: 6808-6820.

[11] P. Yuan, et al. "Very fast hot carrier diffusion in unconstrained MoS 2 on a glass substrate: discovered by picosecond ET-Raman." RSC advances 8(23), 2018, pp: 12767-12778. 